\title{
Research Article \\ Orbital Dynamics of a Simple Solar Photon Thruster
}

\author{
Anna D. Guerman, ${ }^{1}$ Georgi V. Smirnov, ${ }^{2}$ \\ and Maria Cecilia Pereira ${ }^{3}$ \\ ${ }^{1}$ University of Beira Interior, 6201-001 Covilhã, Portugal \\ ${ }^{2}$ University of Porto, 4169-007 Porto, Portugal \\ ${ }^{3}$ Aeronautical Institute of Technology, 12.228-900 Sao José dos Campos, Brazil \\ Correspondence should be addressed to Anna D. Guerman, anna@ubi.pt
}

Received 29 July 2009; Accepted 13 November 2009

Recommended by Maria Zanardi

We study orbital dynamics of a compound solar sail, namely, a Simple Solar Photon Thruster and compare its behavior to that of a common version of sailcraft. To perform this analysis, development of a mathematical model for force created by light reflection on all sailcraft elements is essential. We deduce the equations of sailcraft's motion and compare performance of two schemes of solar propulsion for two test time-optimal control problems of trajectory transfer.

Copyright (C) 2009 Anna D. Guerman et al. This is an open access article distributed under the Creative Commons Attribution License, which permits unrestricted use, distribution, and reproduction in any medium, provided the original work is properly cited.

\section{Introduction}

The use of solar pressure to create propulsion can minimize spacecraft on-board energy consumption during a mission [1, 2]. Modern materials and technologies made this propulsion scheme feasible, and many projects of solar sails are now under development, making solar sail dynamics the subject of numerous studies.

So far, the most extensively studied problem is the orbital maneuver of a Flat Solar Sail (FSS, Figure 1). In this case, the control is performed by turning the entire sail surface with respect to the Sun direction. This changes the radiation pressure and results in evolution of the vehicle trajectory. Some of the many missions studied are described in [3-13].

The use of a compound solar sail, or Solar Photon Thruster (SPT), was proposed by Tsander long ago [1,2], but the study of this spacecraft began quite recently [14-18]. The SPT consists of a parabolic surface which concentrates the solar radiation pressure on a system of smaller mirrors. The control effort in such system is produced by displacement of a small mirror with respect to the parabolic surface. The sail axis is supposed to be oriented along 
the Sun-sailcraft direction. There exist several versions of compound solar sails. Forward [14] described two types of a compound sail, namely, Simple Solar Photon Thruster (SSPT) and Dual Reflection Solar Photon Thruster (DRSPT). The few existent studies on SPT dynamics consider the latter scheme.

In order to assess the dynamical characteristics and to compare the SPT performance to the most studied version, FSS, one should study the application of these propulsion schemes for orbital transfer and/or maintenance for various missions. To perform this analysis, a coherent mathematical model for force acting on such a structure due to solar radiation pressure is essential. There are many results concerning radiation pressure force and torque models for a sunlit body, and in many studies they are applied successively to develop a force model for an FSS. However, the usual approach cannot be applied for a Solar Photon Thruster due to multiple light reflections on the SPT elements.

Some attempts to develop a mathematical description for SPT force have been made before, mostly for a Dual Reflection Solar Photon Thruster. In [2] the model for an ideally reflecting DRSPT is described. In [19] this model is extended for nonideal DRSPT. Meanwhile, both of these models are based on the supposition that all the incoming light flux is reflected consequently on each one of the DRSPT elements and then leaves the system, which is not true [20]. Moreover, the results of [20] show that the existing shadowing and related energy dissipation diminish significantly the DRSPT efficiency, making dubious the advantages of this propulsion scheme compared to FSS.

In the present article, we focus on the other version of the compound scheme of solar propulsion, Simple Solar Photon Thruster (Figure 2). We develop a mathematical model for a solar radiation force acting on SSPT and provide a comparative study of trajectory dynamics and control for the FSS and SSPT schemes. In our analysis, we use the following assumptions.

(i) Solar radiation pressure follows inverse-square variation law.

(ii) The only gravitational field is that of the Sun, and this field is central Newtonian.

(iii) The sails are ideal reflectors (all photons are perfectly reflected).

We derive the equations of motion for the SPT and compare the orbital behavior of FSS and SPT studying two test problems: Earth-Mars transfer and Earth-Venus transfer.

\section{Equations of Motion}

To write down the equations of motion for a solar sail spacecraft, we introduce two rightoriented Cartesian frames with their origin in the center of mass of the spacecraft $O$ as follows:

(i) $O x y z$ is the coordinate frame attached to the spacecraft; the axes $O x, O y$, and $O z$ are the central principal axes of the spacecraft.

(ii) $O X Y Z$ is the orbital frame, its axis $O Z$ is directed along the radius vector of the point $O$ with respect to the center of mass of the Sun, and the axis $O Y$ is orthogonal both to $O Z$ and to the velocity of the point $O$.

We determine the position of the coordinate frame Oxyz attached to the spacecraft with respect to the orbital frame using the transition matrix between these frames, $\left\|a_{i j}\right\|$. 
We use a set of canonical units which implies that the radius of the Earth's orbit is $1 \mathrm{AU}$, and the period of its revolution is $2 \pi$. The equations of orbital motion can be written in the form

$$
\ddot{\vec{r}}=-\frac{\vec{r}}{r^{3}}+\vec{a}_{s}
$$

where $a_{s}$ is the acceleration due to the radiation pressure.

To complete the equations of motion, we have to calculate also the force produced by the Sun radiation pressure.

\subsection{Flat Solar Sail}

The interaction of the solar radiation flow with a flat perfectly reflecting surface has been studied earlier $[1,2]$. By the symmetry of falling and reflected flows, the total solar radiation force is directed along the symmetry axis of the sail and produces no torque with respect to any point of this axis, including the sail's center of mass (Figure 1). This force can be expressed as $[1,2]$

$$
\vec{P}=-2 \vec{n}(\vec{\sigma}, \vec{n})^{2} S \frac{\Phi}{r^{2}}
$$

where $\vec{n}$ is the normal to the sail surface and points to the Sun, $\vec{\sigma}$ is the unit vector of the parallel light flow (i.e., it opposes the Sun-sailcraft direction), its coordinates in the Oxyz frame are $\vec{\sigma}=\left(\sigma_{x}, \sigma_{y}, \sigma_{z}\right)=\left(a_{31}, a_{32}, a_{33}\right), S$ is the total area of the sail, and $\Phi=4.563$. $10^{-6} \mathrm{~N} / \mathrm{m}^{2}$ is the nominal solar radiation pressure constant at $1 \mathrm{AU}$. The solar radiation force projections onto the spacecraft-connected and orbital coordinate frames are

$$
\begin{gathered}
P_{x}=P_{y}=0, \quad P_{z}=2 S \frac{\Phi}{r^{2}} a_{33}^{2} \operatorname{sign} a_{33}, \\
P_{X}=2 S \frac{\Phi}{r^{2}} a_{13} a_{33}^{2} \operatorname{sign} a_{33}, \quad P_{Y}=2 S \frac{\Phi}{r^{2}} a_{32} a_{33}^{2} \operatorname{sign} a_{33}, \quad P_{Z}=2 S \frac{\Phi}{r^{2}}\left|a_{33}\right| a_{33}^{2},
\end{gathered}
$$

respectively.

If $\vec{n}$ lies in the OXZ plane, then the components of the radiation force are

$$
P_{X}=-S \frac{\Phi}{r^{2}} \sin 2 \theta|\cos \theta|, \quad P_{Y}=0, \quad P_{Z}=2 S \frac{\Phi}{r^{2}} \cos ^{2} \theta|\cos \theta|,
$$

where $\theta$ is the angle between the vector $\vec{n}$ and the $O Z$ axis.

The above expressions are standard and appear in numerous studies of the propulsion effort of a Flat Solar Sail. 


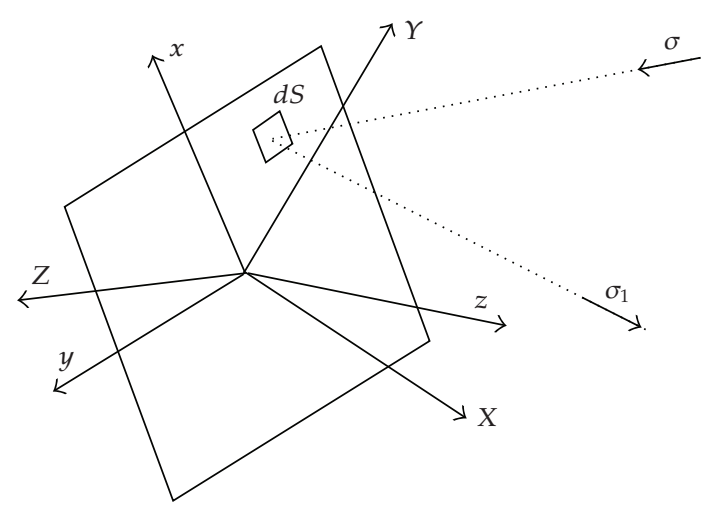

Figure 1: Flat Solar Sail (FSS).

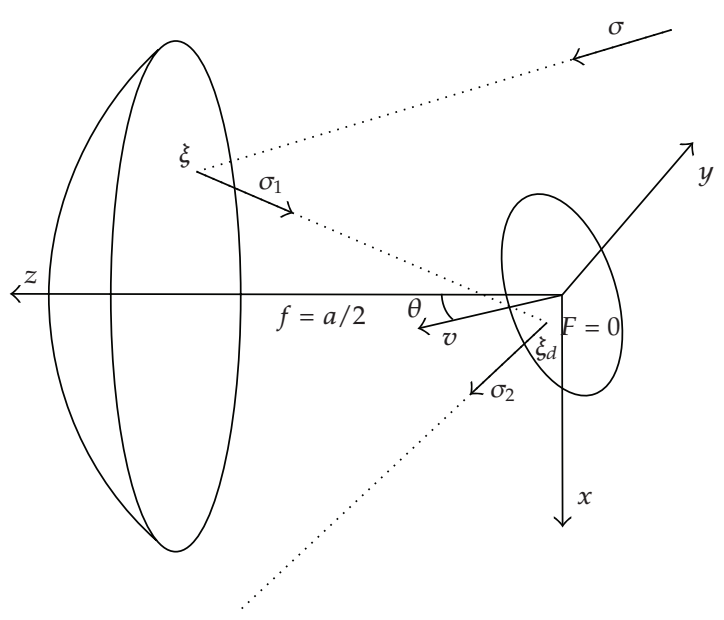

Figure 2: Solar Photon Thruster (SPT).

\subsection{Solar Photon Thruster}

We consider here another system that is shown in Figure 2. It consists of a parabolic collector and a control mirror (director). When reflection is ideal and the collector axis is exactly aligned with the Sun-sailcraft direction, the collector concentrates the sunlight in the center of the director. In order to minimize the solar radiation torque that causes perturbations of the sailcraft orientation, the director should be located at the sailcraft's center of mass. This scheme of solar propulsion seems to be more reliable with respect to small misalignments of the sail axis than the DRSPT scheme studied in [15-17] which uses a collimator.

In the analysis, we assume the control mirror small enough to disregard the influence of its shadow. We also suppose that the SPT axis is aligned exactly along the Sun direction.

We consider the parabolic surface described in the reference frame $O x y z$ by the equation

$$
x^{2}+y^{2}+2 a(z-f)=0, \quad\left(x^{2}+y^{2} \leq R^{2}\right),
$$


where $a$ is the parameter of the paraboloid, $f$ is the focal distance, and $R$ is the radius of the sail's projection on the plane $O x y$. Since $f=a / 2$, equation (2.5) reduces to

$$
z=\frac{1}{2 a}\left(a^{2}-x^{2}-y^{2}\right)
$$

The sunlight is directed along the vector $\vec{\sigma}=(0,0,1)$. Suppose that it is reflected on the element of the parabolic surface $d S$, containing the point $\vec{\xi}=x \vec{i}+y \vec{j}+z \vec{k}$ with $z$ satisfying (2.6). The force produced by the falling light is given by

$$
\overrightarrow{d P}_{1}=-\overrightarrow{\rho \sigma}(\vec{\sigma}, \vec{n}) d S
$$

where $\vec{n}$ is the normal to the sunlit side of the sail surface

$$
\vec{n}=-\frac{x \vec{i}+y \vec{j}+a \vec{k}}{\sqrt{x^{2}+y^{2}+a^{2}}}
$$

and $\rho$ is the intensity of the light flow at the current point of the orbit

$$
\rho=\frac{\Phi}{r^{2}}
$$

The ray reflected from the element $d S$ of the collector's surface has the direction $\vec{\sigma}_{1}$ satisfying

$$
\vec{\sigma}_{1}=\vec{\sigma}-2(\vec{\sigma}, \vec{n}) \vec{n}
$$

Reflection of light from the element of surface $d S$ produces the force

$$
\overrightarrow{d P}_{2}=\overrightarrow{\sigma \sigma}_{1}(\vec{\sigma}, \vec{n}) d S
$$

Finally, this ray is reflected at the focus on the director's surface with the normal $\vec{v}=$ $\left(v_{x}, v_{y}, v_{z}\right)$. The force produced by the reflected light can be written as

$$
\overrightarrow{d P}_{3}=-\overrightarrow{d P}_{2}, \quad \overrightarrow{d P}_{4}=\rho \vec{\sigma}_{2}(\vec{\sigma}, \vec{n}) d S
$$

Here

$$
\vec{\sigma}_{2}=\vec{\sigma}_{1}-2\left(\vec{\sigma}_{1}, \vec{v}\right) \vec{v}
$$

is the direction of the ray reflected from the control mirror. 
The reflection of the light on the parabolic surface is unique if the normal to the director does not cross this surface, so the control angle must be greater than half the angular aperture, that is,

$$
\theta \geq \tan ^{-1}\left(\frac{R}{z_{R}}\right)
$$

Here $\theta$ is the angle between vector $\vec{v}$ and the sail axis $\left(\cos \theta=v_{z}\right)$, and $z_{R}=(1 / 2 a)\left(a^{2}-R^{2}\right)$ is the $z$-coordinate of the collector's border. Finally we arrive at the restriction

$$
|\tan \theta| \geq \frac{2 a R}{a^{2}-R^{2}}
$$

Multiple reflections on the collector destroy the collector's film and produce a considerable disturbing torque, and so have to be avoided. Therefore condition (2.15) has to be satisfied during the orbital maneuver.

The elementary force created by interaction of light with parabolic surface and mirror is

$$
\begin{aligned}
\overrightarrow{d P} & =\overrightarrow{d P}_{1}+\overrightarrow{d P}_{2}+\overrightarrow{d P}_{3}+\overrightarrow{d P}_{4}=\overrightarrow{d P}_{1}+\overrightarrow{d P}_{4} \\
& =-\overrightarrow{\rho \sigma}(\vec{\sigma}, \vec{n}) d S+\rho \vec{\sigma}_{2}(\vec{\sigma}, \vec{n}) d=\rho\left(\vec{\sigma}_{2}-\vec{\sigma}\right)(\vec{\sigma}, \vec{n}) d S .
\end{aligned}
$$

After integration, we obtain

$$
\begin{aligned}
& P_{x}=2 \frac{\Phi}{r^{2}} \pi R^{2} v_{x} v_{z}\left[1-2 \frac{a^{2}}{R^{2}} \ln \left(1+\frac{R^{2}}{a^{2}}\right)\right], \\
& P_{y}=2 \frac{\Phi}{r^{2}} \pi R^{2} v_{y} v_{z}\left[1-2 \frac{a^{2}}{R^{2}} \ln \left(1+\frac{R^{2}}{a^{2}}\right)\right], \\
& P_{z}=2 \frac{\Phi}{r^{2}} \pi R^{2}\left[v_{z}^{2}+\left(1-2 v_{z}^{2}\right) \frac{a^{2}}{R^{2}} \ln \left(1+\frac{R^{2}}{a^{2}}\right)\right] .
\end{aligned}
$$

If the control mirror moves in the OXZ plane $\left(v_{x}=\sin \theta, v_{y}=0, v_{z}=\cos \theta\right)$, then the components of the light pressure force in the orbital coordinate frame are

$$
\begin{gathered}
P_{X}=\frac{\Phi}{r^{2}} \pi R^{2}\left[1-2 \frac{a^{2}}{R^{2}} \ln \left(1+\frac{R^{2}}{a^{2}}\right)\right] \sin 2 \theta, \quad P_{Y}=0, \\
P_{Z}=2 \frac{\Phi}{r^{2}} \pi R^{2}\left[\cos ^{2} \theta-\frac{a^{2}}{R^{2}} \ln \left(1+\frac{R^{2}}{a^{2}}\right) \cos 2 \theta\right] .
\end{gathered}
$$


If $x=R / a \ll 1$, then it is possible to simplify (2.18). One can use Taylor's formula to obtain the expressions

$$
\begin{gathered}
P_{X}=-\frac{\Phi}{r^{2}} \pi R^{2}\left[1-\frac{R^{2}}{a^{2}}+o\left(\frac{R^{3}}{a^{3}}\right)\right] \sin 2 \theta, \quad P_{Y}=0, \\
P_{Z}=2 \frac{\Phi}{r^{2}} \pi R^{2}\left[\sin ^{2} \theta+\frac{R^{2}}{2 a^{2}} \cos 2 \theta+o\left(\frac{R^{3}}{a^{3}}\right)\right] .
\end{gathered}
$$

If $x^{2}=R^{2} / a^{2}$ is negligible (i.e., the sail is almost plane), we get

$$
P_{X}=-\frac{\Phi}{r^{2}} S \sin 2 \theta, \quad P_{Y}=0, \quad P_{Z}=2 \frac{\Phi}{r^{2}} S \sin ^{2} \theta
$$

where $S=\pi R^{2}$ is the effective sail area, that is, the area of the sail projection on the plane $O x y$. Formulas (2.20) are similar to those used in [11-13] for a different scheme of SPT, so one can expect qualitative similarity of the results for small $\chi$, at least for the maneuvers that require control angles within limits (2.15).

\section{In-Plane Orbital Motion}

To compare the principal characteristics of SPT and FSS we studied two test time-optimal control problems of solar sail dynamics, namely, the time-optimal Earth-Mars and EarthVenus transfers $[3,21]$ for both systems. Since our goal is to compare qualitative behavior of the above systems, we choose the simplest formulation for orbital transfer problem. In both cases, we assume that the planet orbits are circular and coplanar and that the spacecraft moves in the ecliptic plane, starting from the Earth-orbit at $1 \mathrm{AU}$ with Earth-orbital velocity. We find the control law that guarantees the fastest transfer to the planet's orbit.

This model of orbital dynamics results in the following equations of motion in the orbit plane [3]:

$$
\dot{r}=u, \quad \dot{\varphi}=\frac{w}{r}, \quad \dot{u}=\frac{w^{2}}{r}-\frac{1}{r^{2}}+a_{s Z}, \quad \dot{w}=-\frac{u w}{r}+a_{s X} .
$$

Here $\varphi$ is the polar angle, and $u$ and $w$ are the radial and transversal components of sail velocity, respectively.

For the FSS, the components of the light pressure acceleration onto the axis of orbital coordinate frame $O X Y Z$ are

$$
a_{S X}=\frac{\Phi}{m r^{2}} S|\cos \theta| \sin 2 \theta, \quad a_{S Z}=2 \frac{\Phi}{m r^{2}} S\left|\cos ^{3} \theta\right|
$$




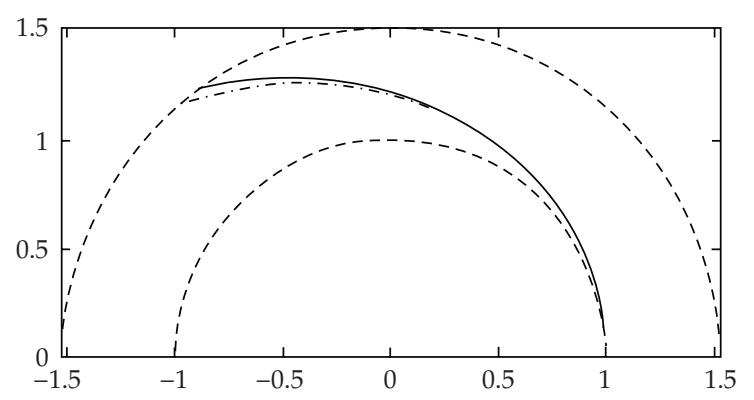

Figure 3: Earth-Mars transfer trajectories.

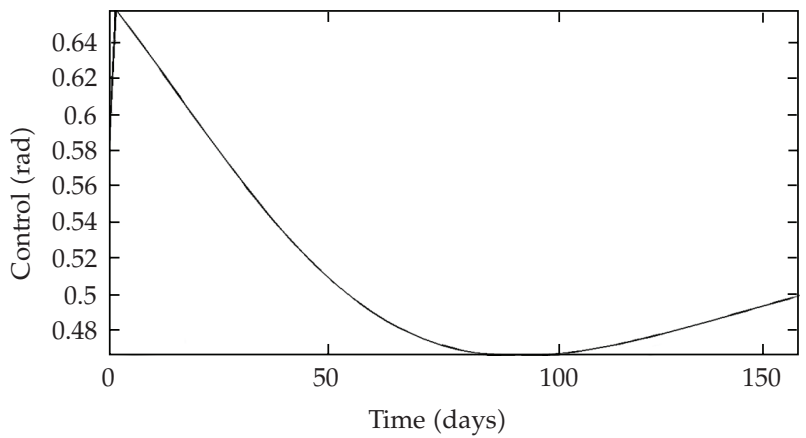

Figure 4: Earth-Mars transfer control effort for SSPT for $\chi=0.125$.

For the SPT the light pressure acceleration is given by

$$
\begin{gathered}
a_{S X}=\frac{\Phi}{m r^{2}} \pi R^{2}\left[1-2 \frac{a^{2}}{R^{2}} \ln \left(1+\frac{R^{2}}{a^{2}}\right)\right] \sin 2 \theta, \\
a_{S Z}=2 \frac{\Phi}{m r^{2}} \pi R^{2}\left[\cos ^{2} \theta-\frac{a^{2}}{R^{2}} \ln \left(1+\frac{R^{2}}{a^{2}}\right) \cos 2 \theta\right] .
\end{gathered}
$$

The control angle $\theta$ is limited by condition (2.15). In this case the sail surface has to follow the Sun direction.

\section{Results}

The time-optimal problems for Earth-Mars transfer and Earth-Venus transfer are studied numerically using the interactive software from [22]. This optimization software developed for personal computers running under MS Windows operating systems is based on the penalty function approach and offers to the user a possibility to effectively solve optimal control problems. During the interactive problem-solving process, the user can change the penalty coefficients, change the precision influencing the stopping rule, and choose/change the optimization algorithms. The system includes various gradient-free algorithms used at 


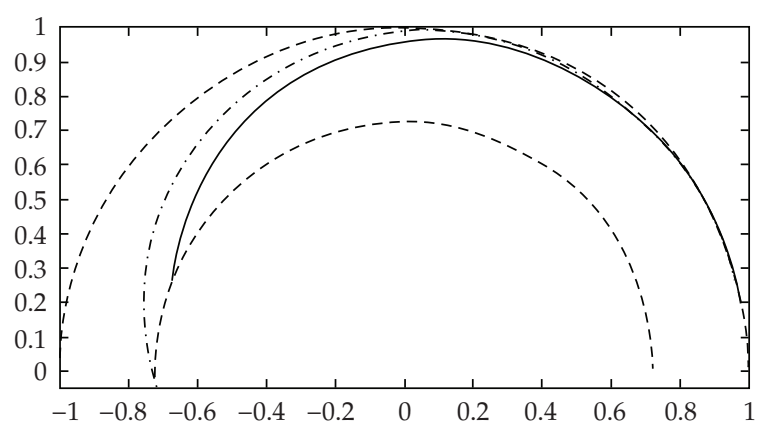

Figure 5: Earth-Venus transfer trajectories.

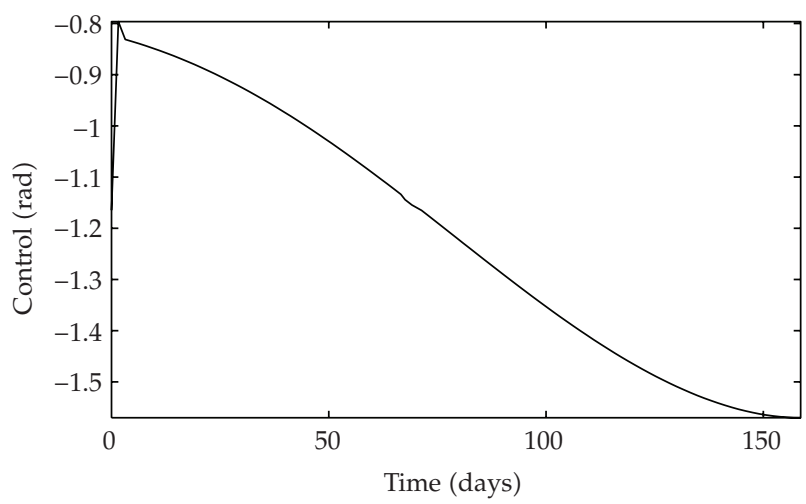

Figure 6: Earth-Venus transfer control effort for SSPT for $x=0.125$.

the beginning of the optimization, as well as more precise conjugate gradient and Newton methods applied at the final stage in order to obtain a precise solution.

The sail parameter is assumed to be $\Phi S / m=0.0843$ for both systems (it corresponds to the acceleration due to solar radiation pressure equal to $1 \mathrm{~mm} / \mathrm{s}^{2}$ at the Earth-orbit).

The Earth-Mars transfer trajectories for the FSS and the SPT are shown in Figure 3. The continuous line corresponds to the SPT trajectory and the dot-dashed line to the FSS trajectory. We consider the ratio $X=R / a=0.125$. The best possible transfer time for the FSS is $T_{\mathrm{FSS}}^{M}=2.87$ (166.7 days), and for the SPT it is $T_{\mathrm{SPT}}^{M}=2.71$ (157.5 days), so SSPT maneuver is $5.6 \%$ faster than that of the FSS one. Figure 4 shows the variation of the SSPT control angle for the optimal transfer; for FSS the respective control is well known [3].

For the Earth-Mars problem, we also study the influence of SPT sail ratio $x$. The increase of $x$ results in longer maneuver time $T_{\mathrm{SPT}}$ : for $\chi=0.25$ it is $T_{\mathrm{SPT}}^{M}=2.76$, and for $x=0.5$ the maneuver time is $T_{\mathrm{SPT}}^{M}=2.96$. For greater values of $x$, the control angle $\theta$ attains the limits described by restriction (2.15) more frequently.

Analyzing the maneuver to Venus orbit for these two sailcraft schemes (Figures 5 and 6), we have established that FSS reaches the objective in 181.2 days $\left(T_{\mathrm{FSS}}^{V}=3.12\right)$, while the SPT performs this maneuver in 158.6 days $\left(T_{\mathrm{SPT}}^{V}=2.73\right)$. In this case, the efficiency of SPT is more significant; SPT reaches Venus orbit $12.5 \%$ faster than FSS. 


\section{Conclusions}

The problems of orbital dynamics and control are studied for two systems of solar propulsion: a Flat Solar Sail (FSS) and a Simple Solar Photon Thruster (SSPT). We develop a mathematical model for force acting on SSPT due to solar radiation pressure, taking into account multiple reflections of the light flux on the sailcraft elements. We derive the SSPT equations of motion. For in-plane motions of an almost flat sail with negligible attitude control errors, these equations are similar to those used in the previous studies of DRSPT.

For these two solar propulsion schemes, FSS and SSPT, we compare the best time response in two test problems (Earth-Mars transfer and Earth-Venus transfer). Our analysis showed a better performance of SSPT in terms of response time. The result was more pronounced for Earth-Venus transfer that can be explained by the greater values of the transversal component of the acceleration developed by SSPT compared to those of FSS.

\section{Acknowledgments}

The authors are grateful to Vladimir Bushenkov for fruitful discussions and his help in use of the optimization software [22]. This work was supported by FCT-Portuguese Foundation for Science and Technology (project CODIS-PTDC/CTE-SPA/64123/2006) and project CoDMoS (Portugal-Brazil collaboration program, FCT-CAPES).

\section{References}

[1] E. H. Polyahova, Space Flight with Solar Sail, Nauka, Moscow, Russia, 1986.

[2] C. R. McInnes, Solar Sailing: Technology, Dynamics and Mission Applications, Praxis Series, Springer, Berlin, Germany, 1999.

[3] B. Ya. Sapunkov, V. A. Egorov, and V. V. Sazonov, "Optimization of Earth-Mars solar sail spacecraft trajectories," Cosmic Research, vol. 30, no. 2, pp. 155-162, 1992.

[4] M. Otten and C. R. McInnes, "Near minimum-time trajectories for solar sails," Journal of Guidance, Control, and Dynamics, vol. 24, no. 3, pp. 632-634, 2001.

[5] G. Colasurdo and L. Casalino, "Optimal control law for interplanetary trajectories with nonideal solar sail," Journal of Spacecraft and Rockets, vol. 40, no. 2, pp. 260-265, 2003.

[6] D. N. Sharma and D. J. Scheeres, "Solar-system escape trajectories using solar sails," Journal of Spacecraft and Rockets, vol. 41, no. 4, pp. 684-687, 2004.

[7] B. Dachwald, "Minimum transfer times for nonperfectly reflecting solar sailcraft," Journal of Spacecraft and Rockets, vol. 41, no. 4, pp. 693-695, 2004.

[8] G. Mengali and A. A. Quarta, "Optimal three-dimensional interplanetary rendezvous using nonideal solar sail," Journal of Guidance, Control, and Dynamics, vol. 28, no. 1, pp. 173-177, 2005.

[9] B. Dachwald, "Optimal solar-sail trajectories for missions to the outer solar system," Journal of Guidance, Control, and Dynamics, vol. 28, no. 6, pp. 1187-1193, 2005.

[10] B. Dachwald, W. Seboldt, and L. Richter, "Multiple rendezvous and sample return missions to nearEarth objects using solar sailcraft," Acta Astronautica, vol. 59, no. 8-11, pp. 768-776, 2006.

[11] M. Macdonald, G. W. Hughes, C. McInnes, A. Lyngvi, P. Falkner, and A. Atzei, "GeoSail: an elegant solar sail demonstration mission," Journal of Spacecraft and Rockets, vol. 44, no. 4, pp. 784-796, 2007.

[12] B. Wie, "Thrust vector control analysis and design for solar-sail spacecraft," Journal of Spacecraft and Rockets, vol. 44, no. 3, pp. 545-557, 2007.

[13] J. Bookless and C. McInnes, "Control of Lagrange point orbits using solar sail propulsion," Acta Astronautica, vol. 62, no. 2-3, pp. 159-176, 2008.

[14] R. L. Forward, "Solar photon thrustor," Journal of Spacecraft and Rockets, vol. 27, no. 4, pp. 411-416, 1990.

[15] C. R. McInnes, "Payload mass fractions for minimum-time trajectories of flat and compound solar sails," Journal of Guidance, Control, and Dynamics, vol. 23, no. 6, pp. 1076-1078, 2000. 
[16] G. Mengali and A. A. Quarta, "Earth escape by ideal sail and solar-photon thrustor spacecraft," Journal of Guidance, Control, and Dynamics, vol. 27, no. 6, pp. 1105-1108, 2004.

[17] G. Mengali and A. A. Quarta, "Time-optimal three-dimensional trajectories for solar photon thruster spacecraft," Journal of Spacecraft and Rockets, vol. 42, no. 2, pp. 379-381, 2005.

[18] B. Dachwald and P. Wurm, "Design concept and modeling of an advanced solar photon thruster," in Proceedings of the 19th AAS/AIAA Space Flight Mechanics Meeting, Savannah, Ga, USA, February 2009, paper no. AAS 09-147.

[19] G. Mengali and A. A. Quarta, "Compound solar sail with optical properties: models and performance," Journal of Spacecraft and Rockets, vol. 43, no. 1, pp. 239-245, 2006.

[20] A. D. Guerman and G. Smirnov, "Comment on "compound solar sail with optical properties: models and performance"," Journal of Spacecraft and Rockets, vol. 44, no. 3, pp. 732-734, 2007.

[21] P. A. Tychina, V. A. Egorov, and V. V. Sazonov, "Optimization of the flight of a spacecraft with a solar sail from Earth to Mars with a perturbation maneuver near Venus," Cosmic Research, vol. 40, no. 3, pp. 255-263, 2002.

[22] G. Smirnov and V. Bushenkov, Curso de Optimização: Programação Matemática, Cálculo de Variações, Controlo Óptimo, Escolar Editora, Lisbon, Portugal, 2005. 


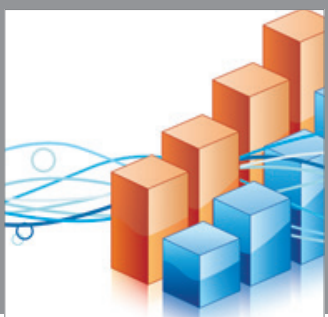

Advances in

Operations Research

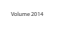

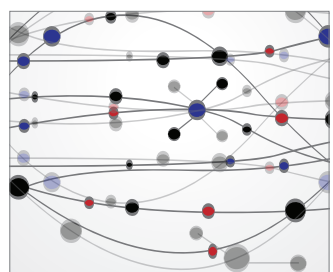

\section{The Scientific} World Journal
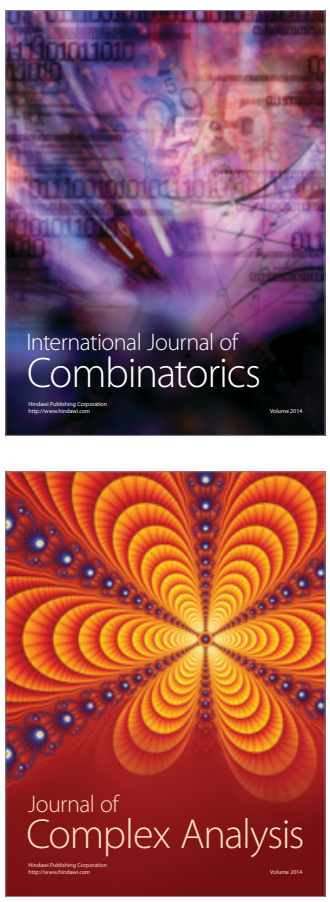

International Journal of

Mathematics and

Mathematical

Sciences
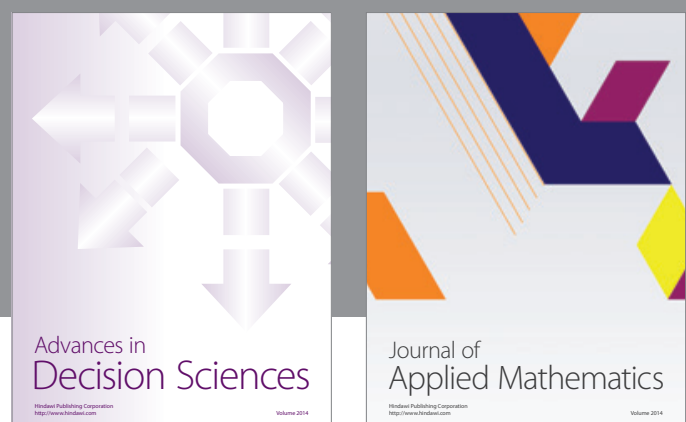

Journal of

Applied Mathematics
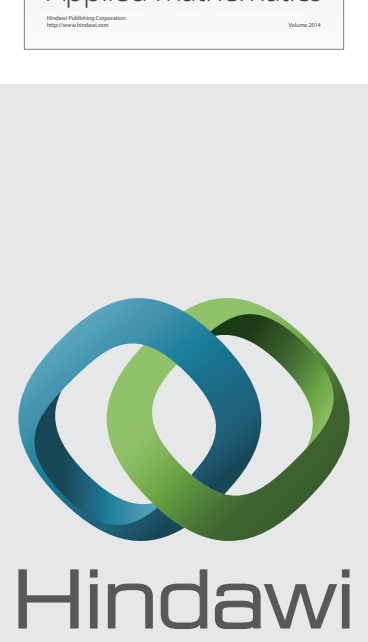

Submit your manuscripts at http://www.hindawi.com
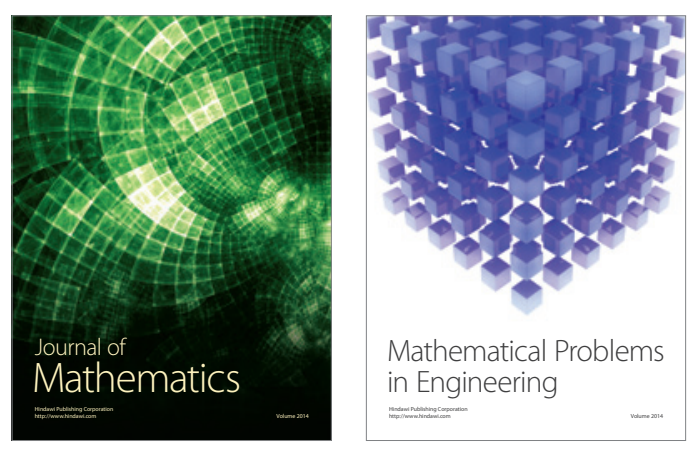

Mathematical Problems in Engineering
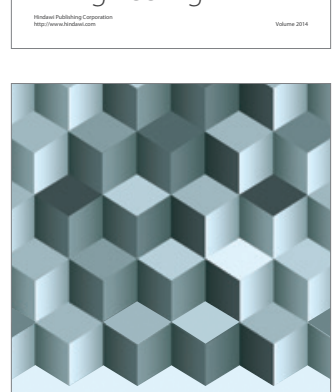

Journal of

Function Spaces
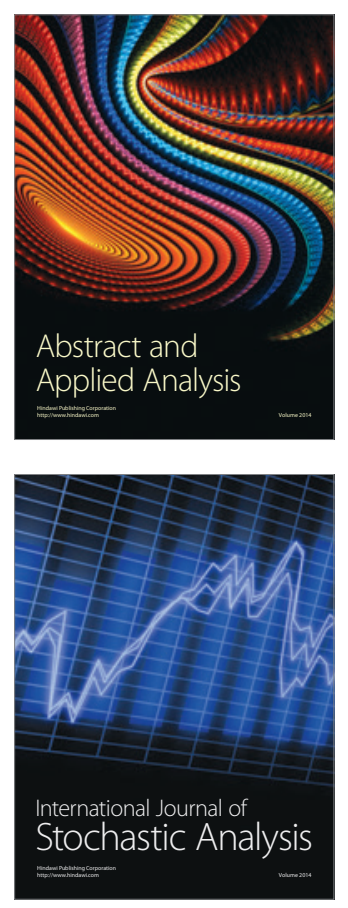

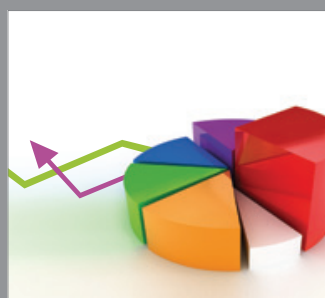

ournal of

Probability and Statistics

Promensencen
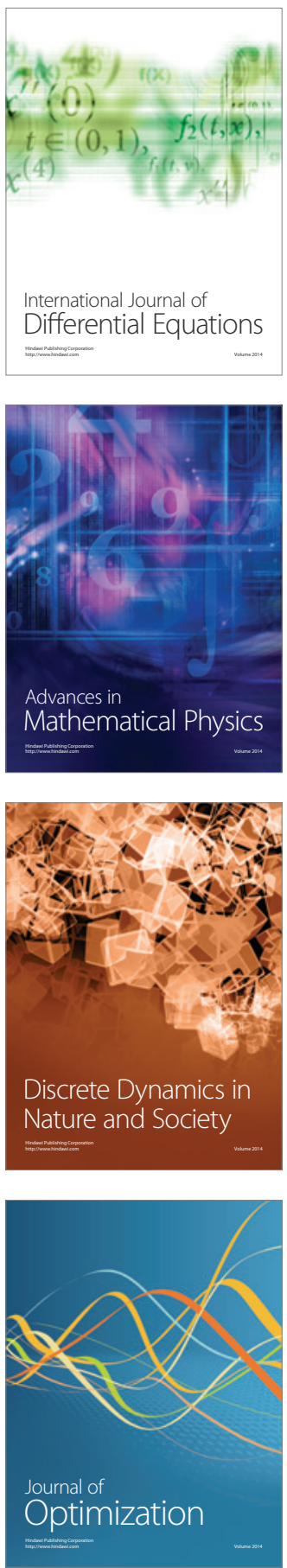